\title{
Cost-effective optical coherence tomography spectrometer based on a tilted fiber Bragg grating
}

\author{
Stefan Remund $^{1 \mathrm{a}}$, Anke Bossen ${ }^{\mathrm{a}}$, Xianfeng Chen ${ }^{\mathrm{b}}$, Ling Wang ${ }^{\mathrm{c}, \mathrm{d}, \mathrm{e}}$, Adedotun Adebayo ${ }^{\mathrm{f}}$, Lin Zhang ${ }^{\mathrm{f}}$, \\ Boris Považay ${ }^{\mathrm{a}}$, Christoph Meier ${ }^{\mathrm{a}}$ \\ ${ }^{a}$ Bern University of Applied Sciences, HuCE OptoLab, BFH-TI, CH-2501 Biel, Switzerland; \\ ${ }^{\mathrm{b}}$ School of Electronic Engineering, Bangor University, Bangor Gwynedd, LL57 1UT, United \\ Kingdom; \\ ${ }^{\mathrm{c}}$ Department of Photoelectric Information Science and Engineering, Jiangnan University, 214122, \\ Wuxi, China; \\ ${ }^{\mathrm{d}}$ Department of Physics and Astronomy, 3001 Heverlee, Belgium; \\ ${ }^{\mathrm{N}} \mathrm{NERF}, 3001$ Heverlee, Belgium; \\ ${ }^{\mathrm{f}}$ Aston Institute of Photonic Technologies, Aston University, Birmingham, B4 7ET, United Kingdom
}

\begin{abstract}
A compact, fiber-based spectrometer for biomedical application utilizing a tilted fiber Bragg grating (TFBG) as integrated dispersive element is demonstrated. Based on a $45^{\circ} \mathrm{UV}$-written PS750 TFBG a refractive spectrometer with $2.06 \mathrm{radiant} / \mu \mathrm{m}$ dispersion and a numerical aperture of 0.1 was set up and tested as integrated detector for an optical coherence tomography (OCT) system. Featuring a $23 \mathrm{~mm}$ long active region at the fiber the spectrum is projected via a cylindrical lens for vertical beam collimation and focused by an achromatic doublet onto the detector array. Covering $740 \mathrm{~nm}$ to $860 \mathrm{~nm}$ the spectrometer was optically connected to a broadband white light interferometer and a wide field scan head and electronically to an acquisition and control computer. Tomograms of ophthalmic and dermal samples obtained by the frequency domain OCT-system were obtained achieving $2.84 \mu \mathrm{m}$ axial and $7.6 \mu \mathrm{m}$ lateral resolution.
\end{abstract}

Keywords: TFBG spectrometer, tilted fiber Bragg grating, optical coherence tomography, miniaturized spectrometer, OCT spectrometer, spectrometer approach, cost-effective spectrometer

\section{INTRODUCTION}

Building spectrometers with diffraction gratings is common practice. Though such spectrometers show high performance, the miniaturization of these devices is limited by the diffraction limit that necessitates expansion and focusing of the beam. Furthermore, the number of components and their long term alignment drives the costs for low maintenance, high resolution instruments. An approach to miniaturize devices and thereby increase their stability is to combine functionalities of different optical components into one, such as with classic concave gratings that integrate the grating with the focusing element [1] or arrayed wave guide gratings that found examples of application in spectrometers for biomedical imaging [2]. These approaches suffer from high complexity of the manufacturing process. A tilted Bragg grating as the dispersive element can be integrated via a rather simple inscription process directly into the optical fiber, which already is an intrinsic component of the optical set-up. The principle realization of a high resolution tilted fiber Bragg-grating (TFBG) Optical Spectrum Analyzer (OSA) was demonstrated [3], [4]. Its simplicity and high potential for efficient light collection makes it appealing for low cost high resolution spectrometry, and spectral imaging techniques like Spectral Domain Optical Coherence Tomography SD-OCT. OCT is a well-established technology to generate noninvasive cross-sectional depth resolved 2-dimensional and 3-dimensional tomograms of biological tissue be measuring the backscattered and back-reflected light [5]-[7] that encodes depth information in a spectral interference signal.

Integration of the spectrometers dispersive element into the fiber eliminates the need for an external diffraction grating and the collimation optics. The reduction of the bulk optical elements increases stability. Together with simple

\footnotetext{
${ }^{1}$ stefan.remund@bfh.ch; optolab.ch
}

Optical Fibers and Sensors for Medical Diagnostics and Treatment Applications XIV, edited by Israel Gannot, Proc. of SPIE Vol. 8938, 89381E · @ 2014 SPIE · CCC code: 1605-7422/14/\$18 · doi: 10.1117/12.2039409 
broadband light sources the miniaturization and simplification results in cost reduction for OCT-devices. This opens new fields of potential applications such as portable OCT devices for flexible and long term monitoring of disease.

\subsection{Tilted Bragg grating fiber}

A tilted Bragg grating fiber (TBG fiber) is an optical fiber with an inscribed fiber Bragg Grating titled at a specific facet angle. The TFBG structure was first demonstrated by Meltz et al. [8] and the theoretical analysis on mode coupling mechanism was given by Erdogan and Sipe [9]. The TFBG alters the propagation direction and causes partial coupling out of the fiber by reflection. The angle of the out-coupled light is strongly wavelength dependent. In other words, adjacent wavelengths, separated by $\delta \lambda$ are separated by the angle $\delta \theta$. The dispersion can be formulated as:

$$
\mathrm{D}=\frac{\delta \Theta}{\delta \lambda}
$$

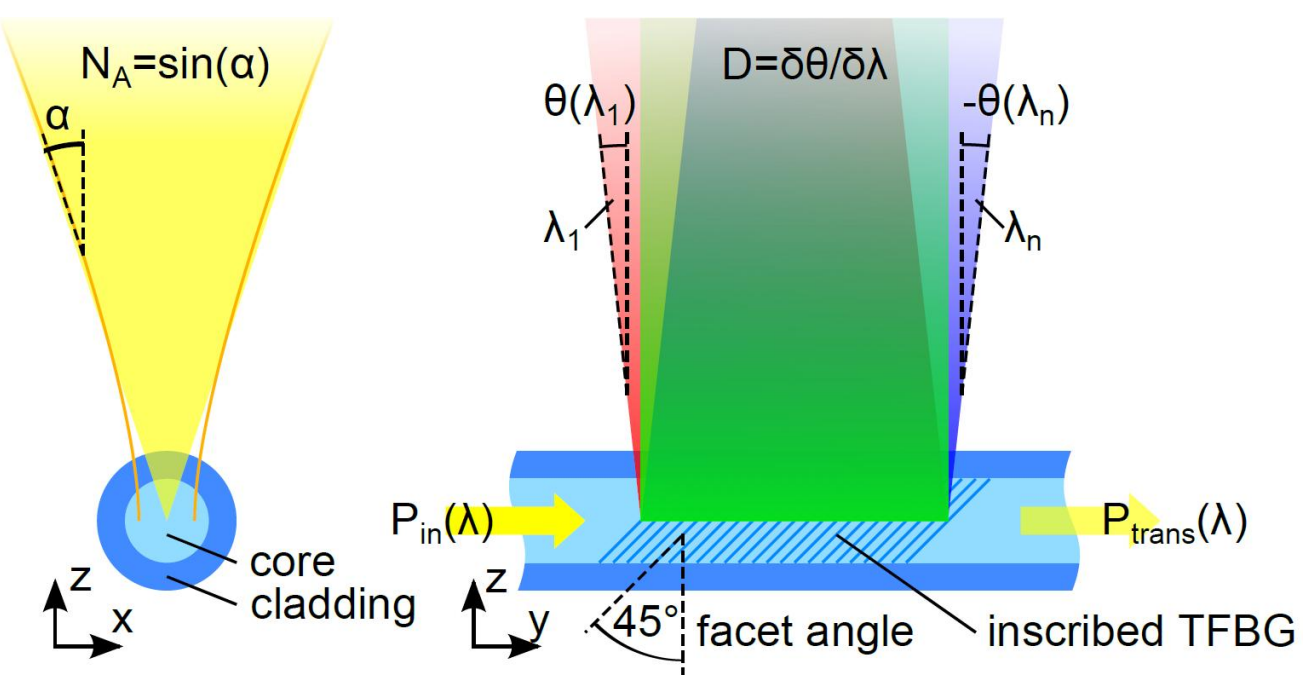

Figure 1. Simplified sketch of a TFBG displaying the emission geometry. Left: Divergence output characteristics in the radial plane of the fiber. Right: Wavelength dependent refraction of the tilted Bragg grating in the axio-lateral plane.

The refraction in the axial direction leads to a diverging light bundle coupling out at angle $\alpha$ in the radial direction, according to the numerical aperture as displayed in Figure 1.

$$
\mathrm{N}_{\mathrm{A}}=\sin (\alpha)
$$

However, only a small portion of the inserted light $\mathrm{P}_{\mathrm{in}}$ is de-coupled by the TFBG. Therefore, a part of the light is not refracted and further guided towards the end of the fiber. These losses $P_{\text {trans }}$ depend on the polarization [9], [10] of the incoming light and the length of the TFBG. As expected the emitted intensity profile in the axial direction follows an exponential decay in propagation direction [11].

\section{SYSTEM DESIGN}

\subsection{Simple two stage spectrometer}

After quantifying the spectral and intensity characteristics of the TFBG in a first stage a simple two-element imaging system was realized, consisting of a cylindrical and a spherical lens of suitable focal lengths. Connected to a high resolution 2D-camera with standard data acquisition and a Michelson interferometer with a motor-driven sample arm the spectral resolution and the corresponding depth dependent fringe loss could be specified as $-16 \mathrm{~dB} / 0.4 \mathrm{~mm}$. Since the sensitivity of this device was limited by the chromatic error of the cylindrical lens, the size of the focusing lens and the instability of the fiber mount a second spectrometer with a USB line-camera (3648 pixels, $8 \mu \mathrm{m}$ pixel pitch) was devised to span a $6.4 \mathrm{~mm}$ depth scan range in air. To optimize the performance of this low-cost configuration the optical concept was revised based on the specifications of the TFBG. 


\subsection{TFBG spectrometer with low cost CCD Line Camera}

A PS750 fiber with an UV-inscribed TBG providing a dispersion of $2.06 \mathrm{radiant} / \mu \mathrm{m}$, a grating length of $23 \mathrm{~mm}$ and a numerical aperture of 0.1 was used to build a spectrometer. For horizontal beam collimation a cylindrical lens 69-747 from Edmund Optics with a focal length of $20 \mathrm{~mm}$ was inserted. The spectral focusing was achieved by an objective consisting of two achromatic doublets AC508-150-B from Thorlabs with focal lengths of $150 \mathrm{~mm}$ using an additional field flattener lens with a focal length of $86 \mathrm{~mm}$ for reducing internal reflections to a low cost USB CCD Line form Mightex with 3648 pixels of the size of $8 \mu \mathrm{m}$ pitch and $200 \mu \mathrm{m}$ height. The field flattener lens was not optimized for this spectrometer design, however, was inserted to reduce the reflection on the uncoated camera glass. The spectrometer was designed for a super luminescence diode laser source (SLED) EBS8000 from Exalos with a central wavelength of $800 \mathrm{~nm}$ the FWHM broadband of $120 \mathrm{~nm}$, which was mapped on approximately 2000 pixels. Since the orthogonally emitted wavelength of the TFBG was $776 \mathrm{~nm}$, the TFBG and the cylindrical lens had to be tilted with respect to the CCD line array. The arrangement of the elements was optimized with the ray trace software Zemax. Figure 2 shows the two different planes as 2D Layout printouts.
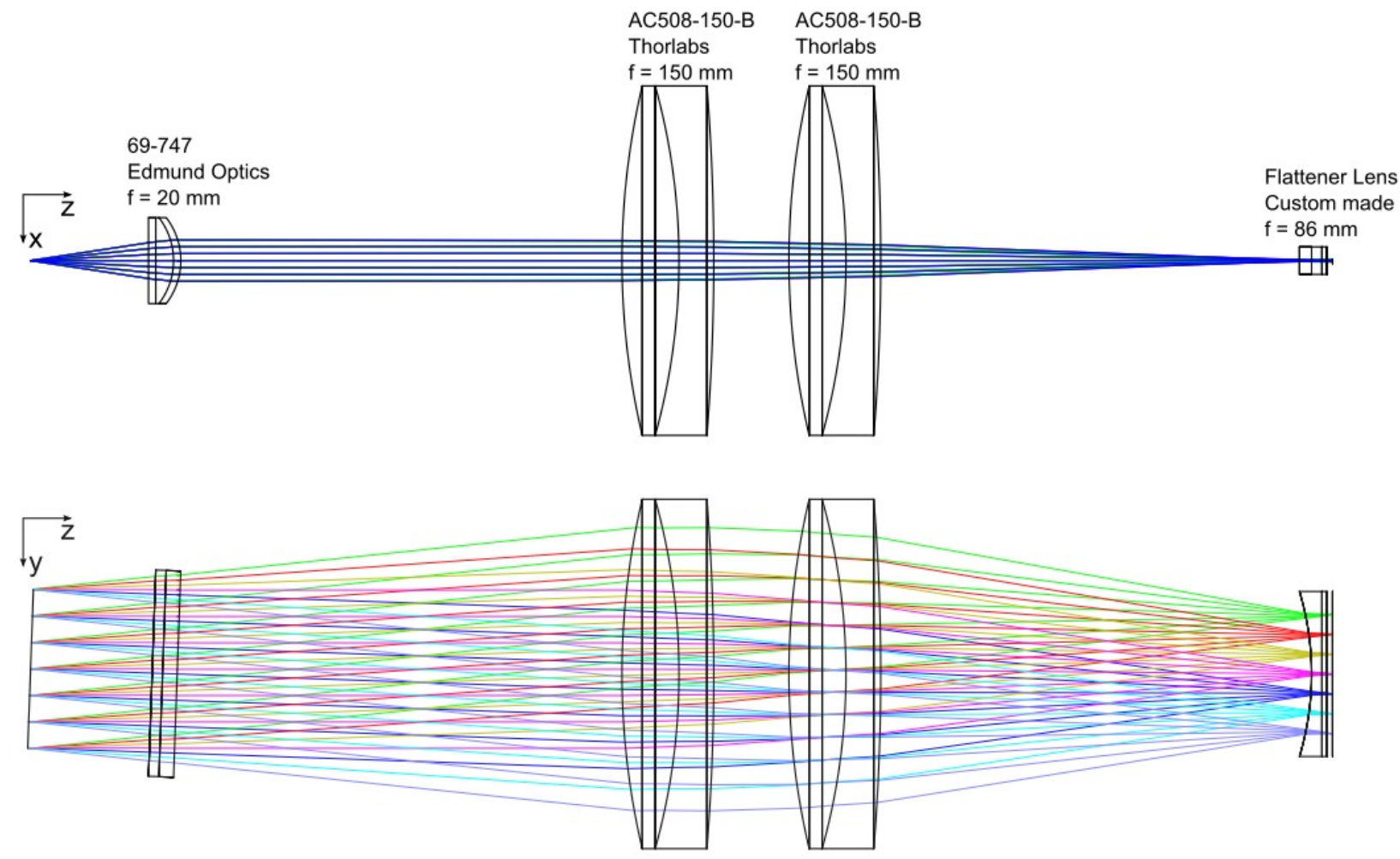

Figure 2. Optical design illustrated in different planes as a printout of the Zemax simulation.

The element positions were numerically optimized for broadband operation to maximize throughput and minimize chromatic error. The $280 \times 80 \times 70 \mathrm{~mm}$ set-up (including the camera) was realized as a cage system supporting the necessary degrees of freedom for the alignment (Figure 3).

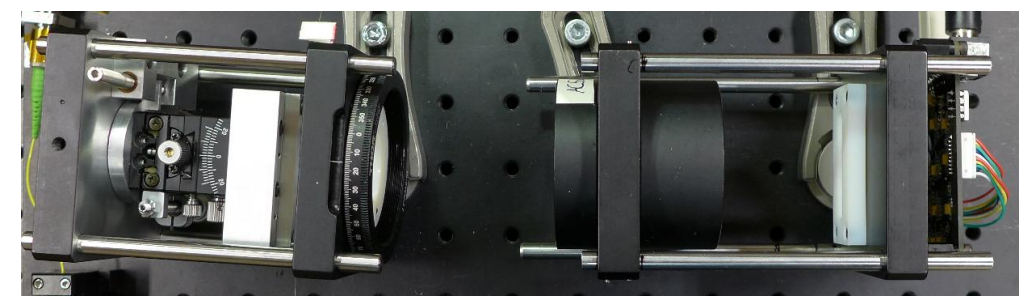

Figure 3. The opto-mechanical design of the TFBG spectrometer 


\subsection{OCT system}

The spectrometer with the TFBG was connected with a common fiber optical Michelson Interferometer OCT setup as schematically shown in Figure 4. The broadband laser light of the SLED $\left(\lambda_{C}=800 \mathrm{~nm}, \Delta \lambda_{\text {FWHM }}=120 \mathrm{~nm}\right)$ is guided in a single mode fiber trough a FC APC plug to a 50:50 fiber coupler where the light is split into reference- and sample arm. The backscattered and reflected light interferes in the fiber coupler before it is analyzed by the TFBG spectrometer. Since the coupling efficiency of the TFBG is highly polarization sensitive, in the reference arm, the sample arm and before the spectrometer, polarization controllers were inserted to adjust the polarization.

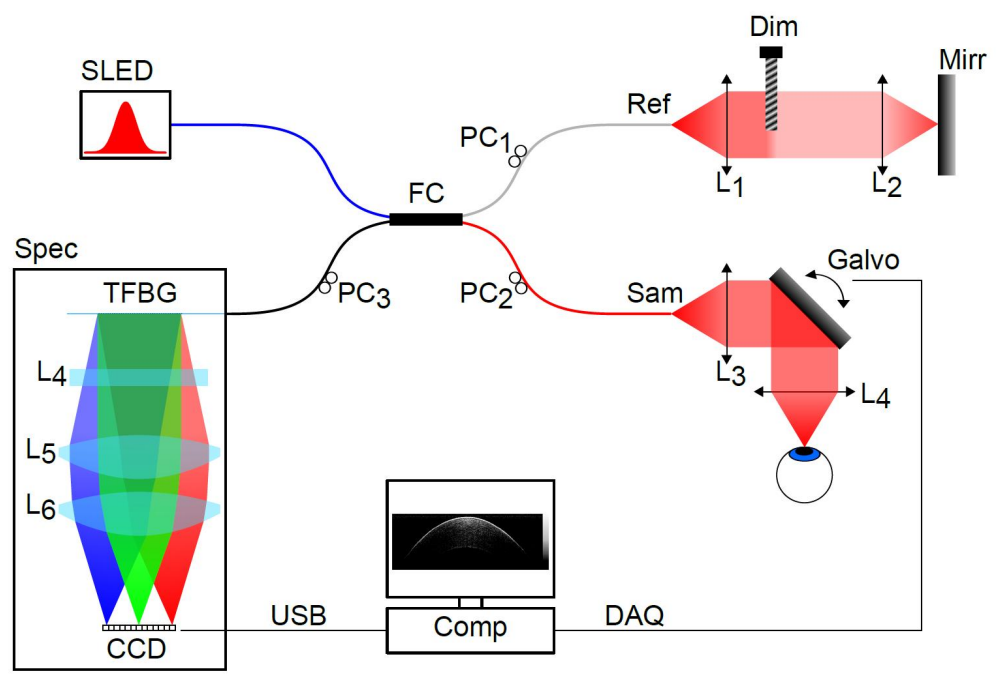

Figure 4. Schematic overview of the OCT system with the implemented TFBG spectrometer: SLED - superluminescence diode, $\mathrm{FC}$ - 50:50 fiber coupler, $\mathrm{PC}_{1}-\mathrm{PC}_{3}-$ Polarization controller, Ref - reference arm, $\mathrm{L}_{1}-\mathrm{L}_{6}-$ lenses, Dim - screw to dim the reference signal, Mirr - silver mirror, Sam - Sample arm, Galvo - galvanometric scanner, Spec - TFBG Spectrometer, TFBG - tilted fiber Bragg grating, CCD - CCD line camera, USB - USB interface, Comp - computer, DAQ - data acquisition cart.

\section{MEASUREMENTS}

The performance of the spectrometer was specified with the OCT system described above. In a first step a sensitivity characterization of the spectrometer was done. In a second step B-Scans of different biological samples were acquired. All measurements were performed with an integration time for the CCD Line Camera of $100 \mu \mathrm{s}$.

\subsection{Sensitivity measurements}

The spectrometer sensitivity can be determined by measuring an extremely low back-reflected signal form the sample arm, calculating the SNR and adding the losses in the sample and the common mode losses from sample arm to the entrance of the spectrometer as Grulkowski et al. describes it in [6]. Therefore the sensitivity can be calculated as follow:

$$
\Sigma=S N R+20 \cdot \log \left(\sqrt{R_{\text {Samp }}} \cdot \sqrt{T_{C M}} \cdot T_{N D}\right)
$$

Where $\mathrm{R}_{\text {Samp }}$ is the reflection of the sample, $\mathrm{T}_{\mathrm{ND}}$ is the transmission of the ND filter inserted in the sample arm to attenuate the signal and $\mathrm{T}_{\mathrm{CM}}$ is the common mode transmission of the sample signal to the spectrometer. The sensitivity and the fall-off of the TFBG spectrometer implemented into the OCT setup are shown in Figure 5. The fall-off of $-34 \mathrm{~dB}$ is caused by the mean RMS (waist) spot size, which is equal to 2.5 times the amount of the pixel pitch. 


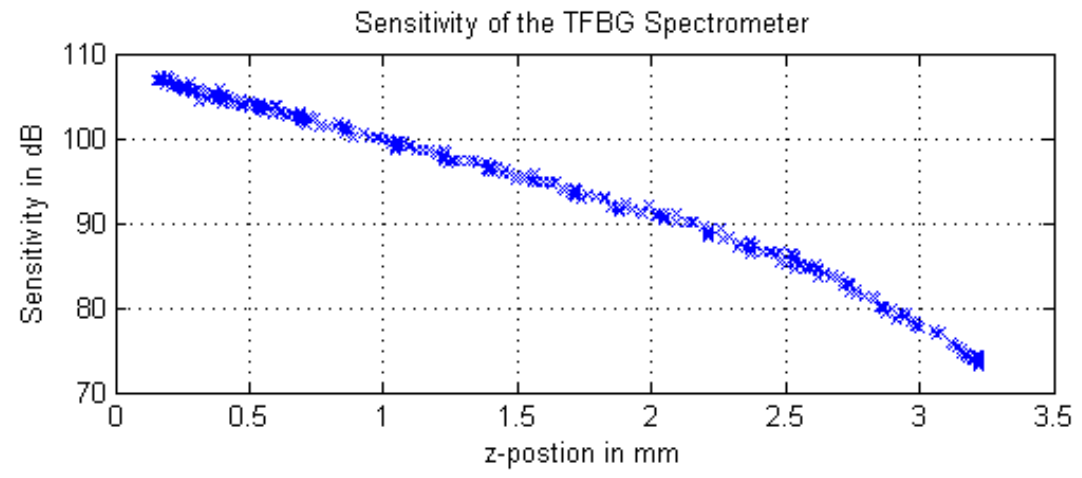

Figure 5. Measured sensitivity over the half depth range, acquired with an integration time of $100 \mu \mathrm{s}$ and an optical power of $0.98 \mathrm{~mW}$ on the sample. The maximum sensitivity reaches $108 \mathrm{~dB}$ and falls-off by $-34 \mathrm{~dB}$ over $3.23 \mathrm{~mm}$.

\subsection{OCT Measurements}

To evaluate imaging performance of the TFBG spectrometer 2-dimensional OCT imaging on a lemon slice shown in Figure 6 was performed. The low scattering and high contrast of the samples cellular structures underlines the high resolution of the device.

Human in-vivo measurements of dermal tissue were obtained by a telecentric 2-D galvonometric scanhead with an effective focal length of $54 \mathrm{~mm}$ and a minimal spot waist of $7.6 \mu \mathrm{m}$. Figure 7 shows a 2-dimensional OCT image of a fingertip. Furthermore ophthalmological cross-sectional OCT images from an anterior segment of a pig eye were acquired (Figure 8). All measurements were performed at an optical power of $0.98 \mathrm{~mW}$ on the sample.

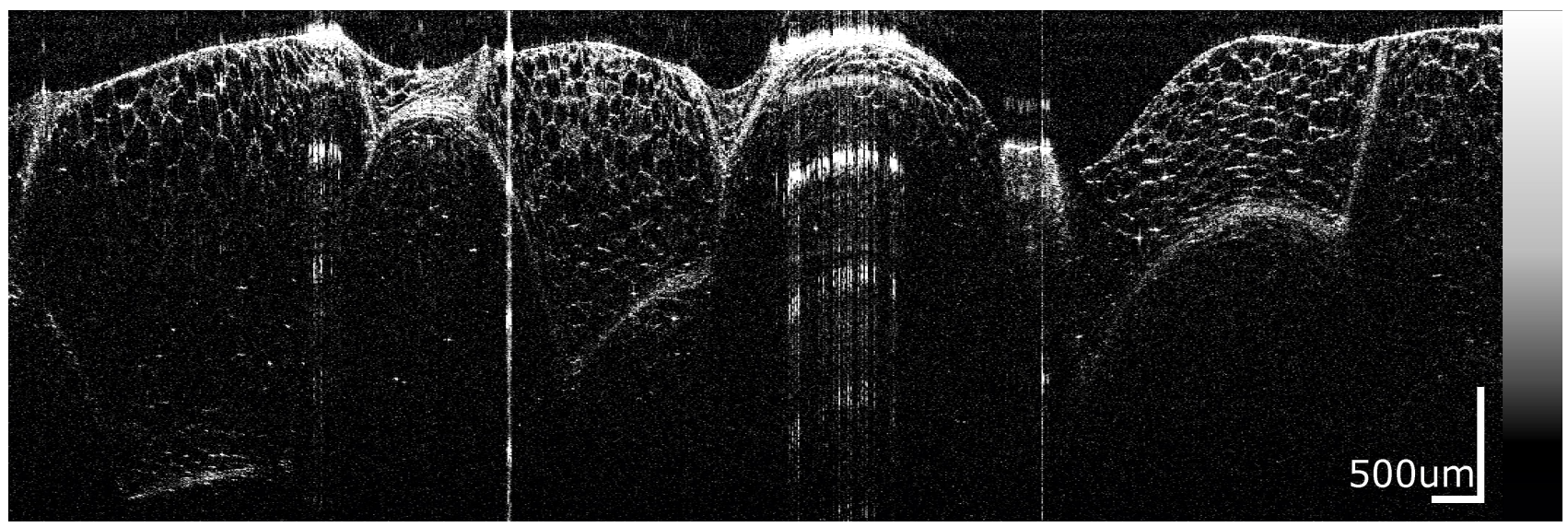

Figure 6. Single tomogram of lemon pulp: lateral scan range of $18 \mathrm{~mm}$, acquired at $100 \mu$ s integration time sampled at $1024 \times 2775$ pixels. 


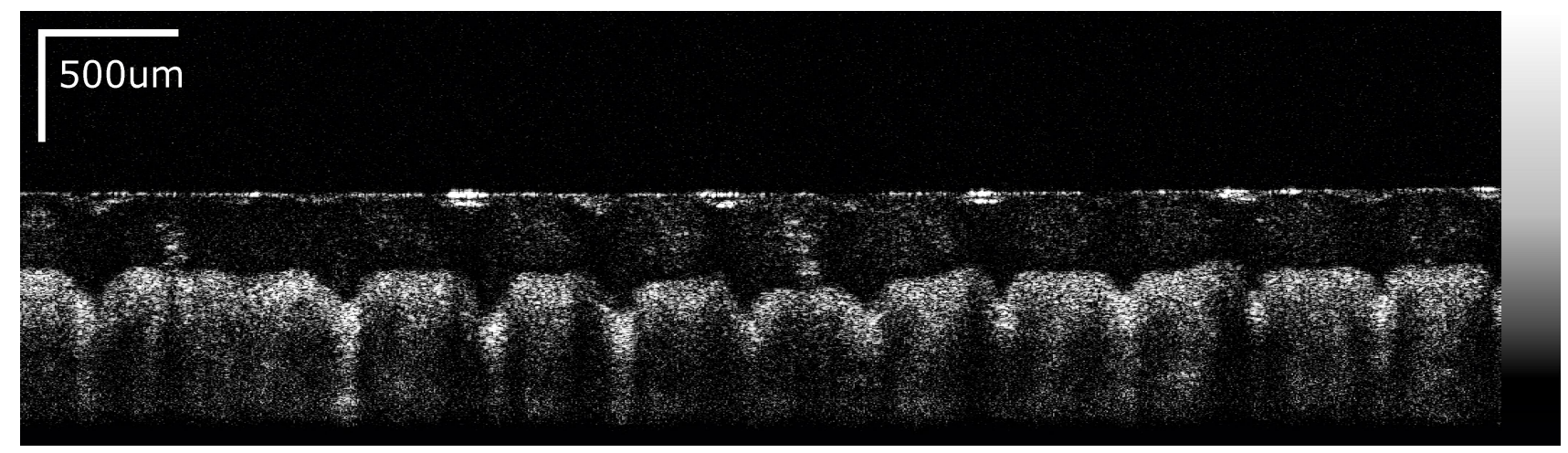

Figure 7. In-vivo OCT image of a fingertip with 1024x1200 pixels from a volunteer acquired on an integration time of $100 \mu \mathrm{s}$ over a scan range of $5.2 \mathrm{~mm}$.
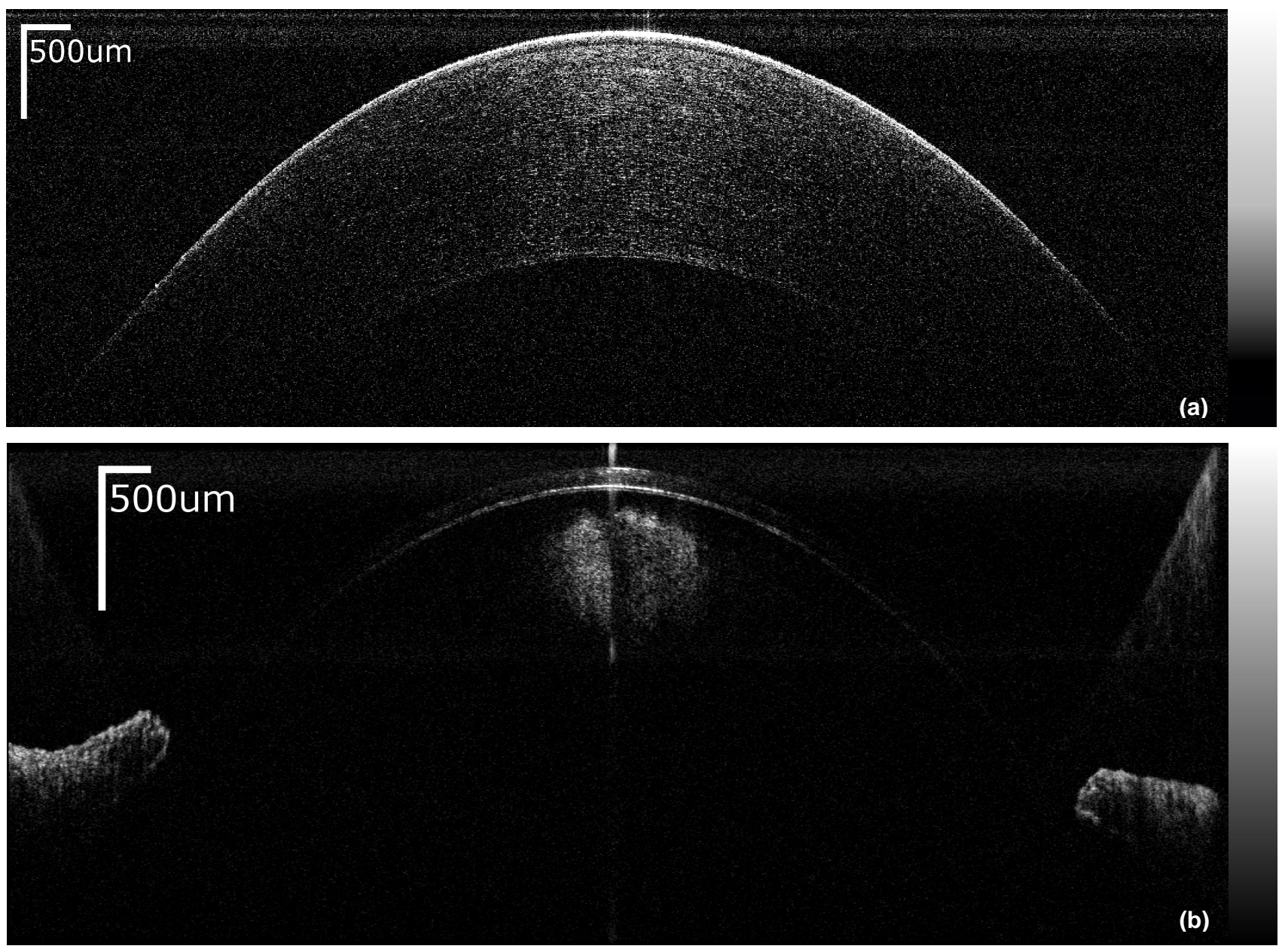

Figure 8. Anterior segment images of a pig-eye made with an integration time of $100 \mu \mathrm{s}$ (a) cross-sectional OCT image of the cornea, scan range of $12.5 \mathrm{~mm}, 1024 \times 1400$ pixels (b) averaging of 18 frames shows lens and the iris, scan range $11.5 \mathrm{~mm}, 667 \times 1096$ pixels. 


\section{CONCLUSION}

The feasibility of a TFBG-spectrometer for SD-OCT was successfully demonstrated. The prototypes maximum sensitivity reaches $108 \mathrm{~dB}$ at $100 \mu$ s integration time and an overall sensitivity fall-off of $-34 \mathrm{~dB}$ across $6.4 \mathrm{~mm}$ detection length. Cross-sectional 2-D OCT images of a fruit, a human fingertip and the anterior segment of a pig-eye were demonstrated and depict a reasonable image quality for this preliminary low cost system that is well comparable to competing devices.

Further improvement of the optical design and the TFBG fabrication process have potential to optimize the TFBG spectrometer towards smaller size and higher efficiency to achieve better optical performance and higher imaging speed. In the current setup the detection probe is the limiting component rather than the detection side. Even without further optical optimization the device can be fitted into a $200 \times 60 \times 10 \mathrm{~mm}$ format after removal of unnecessary optical surfaces using only standard components. To reduce the depth dependent signal loss which is caused by the limited spectral resolution an increase of the numerical aperture is necessary. This is usually associated with enlarged optics. A growth of the device can be counteracted by a more efficient grating with shorter emission length, but higher coupling ratio, so that the radii of curvature can be reduced. Similar to concave gratings also reflective designs are possible. Here, however, the integration of the dispersive component into the fiber instead of the reflector does not necessitate complex manufacturing processes for blazed surfaces to achieve high efficiency.

The relatively simple design and dense integration as a fiber-optic adapter to an existing cheap optical-electronic infrastructure overcomes mechanic limitations of bulk systems, enables miniaturization at reduced costs and has the potential to extend the field of application for OCT-systems in biology, medicine and technology.

\section{ACKNOWLEDGEMENTS}

The authors thank Michael Peyer for his support in image processing, Hansjörg Hegg for precise manufacturing of the mechanical elements, Dominic Krakowski from Mightex Systems for his support with the camera hardware and Thomas Lüthi, Dominic Ernst and Deborah Chansorn for the profitable discussions.

\section{REFERENCES}

[1] Loewen, E. G. and Popov, E., [Diffraction Gratings and Applications], CRC Press, 1997.

[2] Akca, B. I., Považay, B., Alex, A., Wörhoff, K., de Ridder, R. M., Drexler, W., and Pollnau, M., "Miniature spectrometer and beam splitter for an optical coherence tomography on a silicon chip," Optics Express, vol. 21, no. 14, p. 16648, Jul. 2013.

[3] Wagener, J. L., Strasser, T. A., Pedrazzani, J. R., DeMarco, J. and DiGiovanni, D. J., "Fiber grating optical spectrum analyzer tap," in Integrated Optics and Optical Fibre Communications, 11th International Conference on, and 23rd European Conference on Optical Communications (Conf. Publ. No.: 448), 1997, vol. 5, pp. 65-68 vol.5.

[4] Zhou, K., Cheng, X., Yan, Z., Adedotun, A. and Zhang, L., "Optical Spectrum Analyzer using a $45^{\circ}$ tilted fiber grating," Advanced Photonics Congress, 2012, p. BW2E.7.

[5] Huang, D., Swanson, E. A., Lin, C. P., Schuman, J. S., Stinson, W. G., Chang, W., Hee, M. R., Flotte, T., Gregory, K., Puliafito, C. A. and Fujimoto, J. G., "Optical coherence tomography," Science, vol. 254, no. 5035, pp. 11781181, Nov. 1991.

[6] Grulkowski, I., Gora, M., Szkulmowski, M., Gorczynska, I., Szlag, D., Marcos, S., Kowalczyk, A. and Wojtkowski, M., “Anterior segment imaging with Spectral OCT system using a high-speed CMOS camera," Opt. Express, vol. 17, no. 6, pp. 4842-4858, Mar. 2009.

[7] Fercher, A. F., "Optical coherence tomography - development, principles, applications," Zeitschrift für Medizinische Physik, vol. 20, no. 4, pp. 251-276, Nov. 2010.

[8] Meltz, G., Morey, W. W. and Glenn, W. H., "In-fiber Bragg grating tap," Optical Fiber Communication, 1990, p. TUG1.

[9] Erdogan, T. and Sipe, J. E., "Tilted fiber phase gratings," J. Opt. Soc. Am. A, vol. 13, no. 2, pp. 296-313, Feb. 1996. 
[10] Zhou, K., Simpson, G., Chen, X., Zhang, L. and Bennion, I., "High extinction ratio in-fiber polarizers based on $45^{\circ}$ tilted fiber Bragg gratings," Opt. Lett., vol. 30, no. 11, pp. 1285-1287, Jun. 2005.

[11] Adebayo, A., Yan, Z., Zhou, K., Zhang, L., Fu, H. and Robinson, D., "Power Tapping Function in Near Infra-Red Region Based on $45^{\circ}$ Tilted Fiber Gratings,” Optics and Photonics Journal, vol. 03, no. 02, pp. 158-162, 2013. 